\title{
Comment on the article "Optimal port site placement" by R. W. Stitz
}

\author{
F. Konishi
}

Received: 12 June 2010/Accepted: 12 June 2010/Published online: 17 August 2010

(C) Springer-Verlag 2010

Port placement is important in laparoscopic colorectal surgery. In Japan, as well as in overseas countries, the pattern of port placement varies from surgeon to surgeon. Having read the article by Professor Russell Stitz [1], I will give some of my comments on port placement in laparoscopic colorectal surgery based on my experience in Japan. Professor Stitz mentioned that in anterior resection of the sigmoid and the rectum, it is usual to mobilize the splenic flexure. However, because of the long sigmoid colon often found in Japanese patients, in about $80 \%$ of sigmoid or rectal cancer patients, there is no need to mobilize the splenic flexure. Because of this, port placement for surgery of the sigmoid and the rectum in Japanese patients is somewhat different from that of Professor Russell Stitz. The second point I would like to make is that Japanese surgeons are keen on lymph node dissection up to the origins of the mesenteric arteries. This is especially true in the case of right sided colon cancer when we usually expose the trunk of the superior mesenteric vein and the origins of the ileocolic, right colic, gastrocolic (vein) and middle colic vessels depending on the site of the tumor. In order to perform such a meticulous lymph node dissection, an operator usually stands between the patient's legs and uses trocars placed on both sides of the lower abdomen. As for the types of instrument, I agree that bladeless trocars cause less trauma and are far better than those with blades. However, they are also more expensive. In most of the laparoscopic colorectal surgery procedures in Japan, it is

F. Konishi $(\bowtie)$

Department of Surgery, Saitama Medical Center, Jichi Medical University, 1-847 Amanuma-cho, Omiya-ku, Saitama, Japan e-mail: dzd00740@nifty.ne.jp standard practice to place a Hasson-type trocar below the umbilicus. Although the site of the small incision for specimen extraction varies among Japanese laparoscopic colorectal surgeons, the initial camera port is almost always the Hasson type, and is placed below the umbilicus, or at the umbilicus except in patients with adhesions. The following are the port positions for anterior resection and right colectomy in my practice.

In anterior resection of the sigmoid and the rectum (Fig. 1), a $12 \mathrm{~mm}$ trocar is placed in the right lower quadrant (A). This is used for the insertion of a linear stapler. Some Japanese laparoscopic surgeons insert the port for the linear stapler suprapubically at the midline. I prefer to place it in the lower right quadrant. In the case of rectal cancer, this port should be placed as low as possible. As Professor Stitz mentioned, we always identify the right inferior epigastric artery by laparoscopic observation which can be done easily with a $0^{\circ}$ scope. A $10 \mathrm{~mm}$ port is placed at the left lower quadrant (B) (Fig. 1). The reason for using a $10 \mathrm{~mm}$ rather than a $5 \mathrm{~mm}$ port is that we make a small incision for specimen extraction at the site of the left lower quadrant trocar. A $5 \mathrm{~mm}$ trocar is placed on the left side above the level of the umbilicus (C) (Fig. 1). In a simple sigmoid cancer operation, these four ports are sufficient. But in cases of obesity or rectal tumors we usually place an additional trocar on the right side above the level of the umbilicus (D) (Fig. 1). Port sites A and C or A and D will be used by the operator, and port site $\mathrm{B}$ or $\mathrm{B}$ and $\mathrm{C}$ will be used by the assistant for traction of the mesentery and/or the rectum. When splenic flexure mobilization is anticipated, port site $\mathrm{B}$ should be placed more caudally to facilitate the dissection around the splenic flexure.

In right colectomy (Fig. 2), in addition to the periumbilical Hasson port, a $5 \mathrm{~mm}$ port (A) is placed in the right lower quadrant, a $10 \mathrm{~mm}$ port (B) is placed in the left lower 


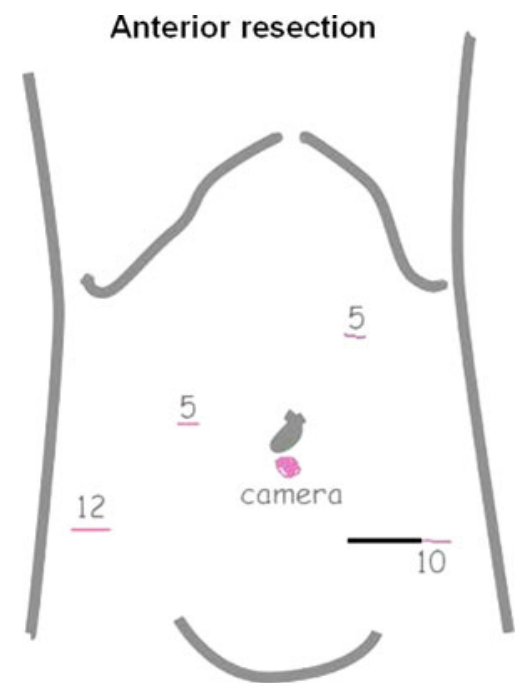

Fig. 1 Port placement for laparoscopic anterior resection

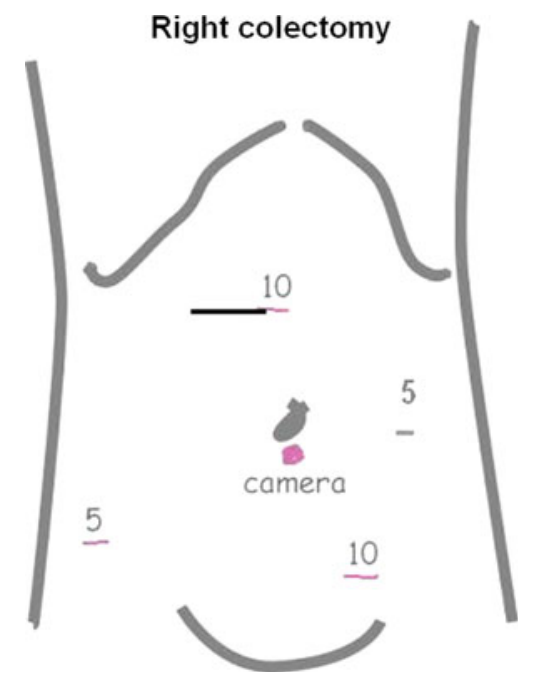

Fig. 2 Port placement for laparoscopic right hemicolectomy

quadrant (usually medial to the left epigastric vessels), and a $10 \mathrm{~mm}$ port is placed above and slightly to the right of the umbilicus (C). An additional $5 \mathrm{~mm}$ port may be placed to the left of the umbilicus (D) (Fig. 2). For the dissection of the right mesentery and the lymph node dissection exposing the superior mesenteric vein and its branches, the operator uses port sites $\mathrm{A}$ and $\mathrm{B}$, and the assistant uses port sites $C$ and $D$ (if necessary) for traction of the right colon (or transverse colon) and its mesentery. The reason for choosing a $10 \mathrm{~mm}$ trocar for port site B is that it will allow the use of small gauze swabs to remove blood during dissection. A small incision for specimen extraction is made at the site of port $\mathrm{C}$, which is the reason for using a $10 \mathrm{~mm}$ port at this site.

I understand very well the reasons for Professor Russel Stitz's recommendations for port placement in different procedures of laparoscopic colorectal surgery. There are probably some other variations in the world including our Japanese method.

\section{Reference}

1. Stitz RW (2010) Optimal port site placement. Tech Coloproctol 14. doi:10.1007/s10151-010-0595-y 